\title{
Adding muscle to neuromuscular degeneration investigation
}

btaining accurate muscle degeneration data for neuromuscular diseases such as amyotrophic lateral sclerosis, muscular dystrophy, inflammatory muscle diseases, and other related disorders is difficult, as the standard tests available are painful and/or subjective.

A new, noninvasive method, called electrical impedance myography (EIM), which is now undergoing detailed testing in patients, holds promise as an accurate and painless means to assess muscle tissue characteristics. EIM takes advantage of a fundamental trait of skeletal muscle in order to measure tissue degeneration. Muscle tissue, made up of a series of tightly bundled fibers, is not perfectly cylindrical but rather is thicker in some areas than others; thus, when passing through it, an electrical current must flow both parallel and transverse to the bundles to reach all parts of the tissue. The transverse current passes through a series of cell membranes, and in healthy cells, these act as insulators and delay the current. The difference between the two currents is called phase: the shorter the delay, the lower the phase and the more diseased the tissue.

Ronald Aaron and Carl Shiffman, professors in the Department of Physics at Northeastern University, Boston, have worked on EIM since the mid-1990s. Shiffman told the JCI, "The reason we got into it [EIM] was because we really wanted to do measurements on the head. But we had never done any measurements on people, and we were a bit scared about sticking electrodes in people's heads and pushing currents in. So we said, 'Why don't we try the thigh?' . . . Little by little, ... we saw there were some socalled normal people who had very low phases, and when we inquired further, we found they weren't normal: they had had a heart attack recently... But [turn-

EIM data. Resistance measurement in a lower-leg muscle (the bilateral tibialis anterior) of a male with a right L4-5 radiculopathy and weak foot dorsiflexion. The patient's left leg is normal.

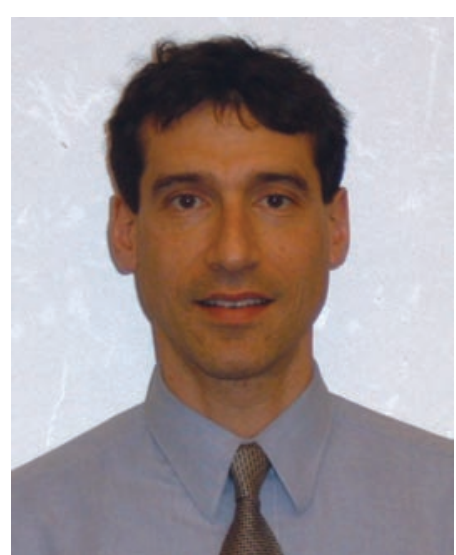

Seward Rutkove cautioned that EIM currently is a work very much in progress.

ing this into] a systematic approach to [test for] muscle disease didn't occur until Seward [Rutkove] contacted us.”

Rutkove, a professor in the Department of Neurology at Beth Israel Deaconess Medical Center, picked up the thread of the story. "I was in the neuromuscular division and seeing a lot of patients with different nerve and muscle diseases. The standard way of testing is called electrical myography. It is uncomfortable and invasive. I had kept thinking, 'Can't we take a different approach?' So I started reading the literature." It took him a while, but eventually he found some articles by Aaron and Shiffman. Rutkove said that when he read their work, he thought, "We just need to apply this to neuromuscular disease, and we might actually see something interesting.' Then I said, 'Oh, they

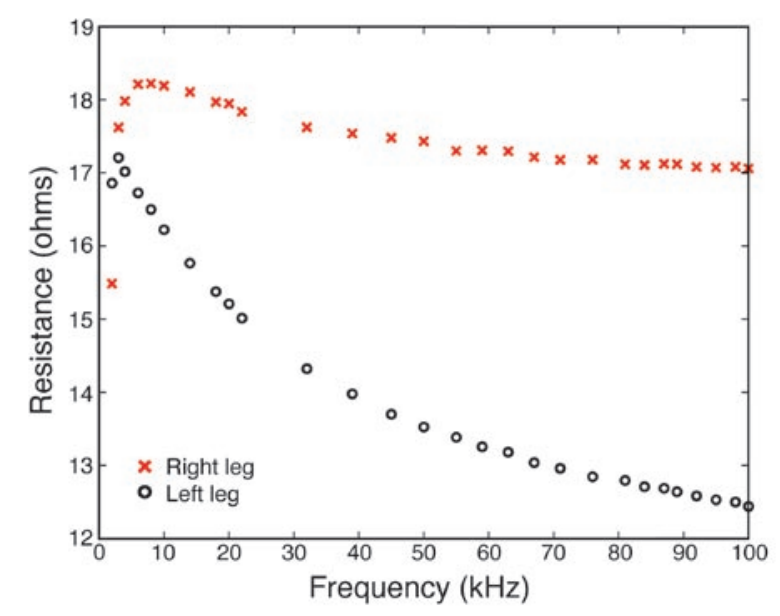

probably live in California or something.' But I looked at their location, and they happen to be across the street, practically. It was kind of a fun coincidence."

The consequences of these serendipitous circumstances may end up benefitting neuromuscular patients and researchers. The initial tests on the usefulness of EIM have been encouraging. For example, a "ROC test" - which stands for "receiver operating characteristic analysis" and is a widely used threshold-independent test in clinical medicine to assess a method's sensitivity and selectivity - has already been done using EIM and was very successful.

"If the [ROC] score is above about 0.9 , it is considered a useful test. Our data were 0.95 ," Shiffman explained. Their study included 45 healthy people and 25 with a neuromuscular disease. The NIH has since provided a grant for more extensive testing. Aaron, Shiffman, and Rutkove have already recruited another 80-90 people and are aiming for a total of about 350: 110 healthy and 240 with various neuromuscular diseases. They will be assessing the usefulness of many aspects of EIM beyond the measurement of phase, including the direct use of the raw data that goes into the phase calculation, such as reactance and resistance. Their first goal will be reestablishing the previous results on a larger population, but they hope they will ultimately be able to detect EIM patterns specific to various disease types.

All three remain cautious about this technology, emphasizing that EIM is still in development. But they are cautiously optimistic. Shiffman noted that they have been following several patients over time, including one ALS patient for about four years. Shiffman told the JCI, "This is an outstanding case in the sense that when we first measured the properties of his thigh, he looked perfectly normal. The disease had struck his fingers and forearms but hadn't progressed to his thighs yet. But in a month or so, we started to pick it up in the thigh, and then there was a very pronounced, steady, almost linear drop [in phase] with time."

Aaron, Shiffman, and Rutkove anticipate that the next phase of their EIM studies, if nothing else, will prove to be stimulating. 